\title{
CONTINUOUS MAPPINGS FROM CANTOR SPACES ONTO INVERSE LIMIT SPECTRA
}

\author{
ALAN H. SCHOENFELD
}

\begin{abstract}
Let $\delta=\left\{X_{\alpha} ; f_{\alpha \beta}: X_{\beta} \rightarrow X_{\alpha}\right\}$ be an inverse limit spectrum of compact Hausdorff spaces. We obtain necessary and sufficient conditions that there be a closed subspace $W$ of a Cantor space and a family $\left\{f_{\alpha}: W \rightarrow X_{\alpha}\right\}$ of continuous surjections such that for each pair $\alpha<\beta$, $f_{\alpha \beta} \circ f_{\beta}=f_{\alpha}$. This result is applied to a special class of inverse spectra.
\end{abstract}

I. Introduction. It is well known that any compact Hausdorff space is the continuous image of a closed subspace of a Cantor space, any compact metric space the continuous image of $2^{\omega}$, the Cantor set. This paper was motivated by the following problem:

Let $\left\{X_{i} \mid i=0,1,2\right\}$ be compact metric spaces and let $\left\{f_{0 i}: X_{i} \rightarrow X_{0} \mid i=1,2\right\}$ be given continuous surjections. Do there exist continuous surjections $\left\{f_{i}: 2^{\omega} \rightarrow X_{i} \mid i=0,1,2\right\}$ such that $f_{0}=f_{01} \circ f_{1}=f_{02} \circ f_{2}$ ?

The answer (affirmative) follows easily from Theorem 3. Since the collections $\left\{X_{i} ; f_{i j}\right\}$ form a (trivial) inverse spectrum, that result suggested that one might examine families of maps onto inverse spectra in general.

AGREEMENT. In this paper we assume each space $X_{\alpha}$ is nonempty, and that the bonding map $f_{\alpha \alpha}: X_{\alpha} \rightarrow X_{\alpha}$ is the identity on $X_{\alpha}$ for each $\alpha$. The $\alpha$ th projection from $\prod_{\alpha} X_{\alpha}$ to $X_{\alpha}$ will be denoted $p_{\alpha}$. We will denote $\operatorname{Lim} X_{\alpha}$ by $X_{\infty}$.

\section{General maps onto inverse limit spectra.}

Definition 1. Let $\delta=\left\{X_{\alpha} ; f_{\alpha \beta}: X_{\beta} \rightarrow X_{\alpha}\right\}$ be an inverse spectrum. Let $S$ be a set and $\left\{f_{\alpha}: S \rightarrow X_{\alpha}\right\}$ a collection of set maps such that

(i) Each $f_{\alpha}$ is a surjection.

(ii) If $\alpha<\beta, f_{\alpha}=f_{\alpha \beta} \circ f_{\beta}$.

Then we say the $\left\{f_{\alpha}\right\}$ map $S$ onto $\delta$.

THEOREM 1. Let $\delta=\left\{X_{\alpha} ; f_{\alpha \beta}: X_{\beta} \rightarrow X_{\alpha}\right\}$ be an inverse spectrum of compact Hausdorff spaces. Then (A) and (B) below are equivalent.

(A) There is a set $S$ and a family of set maps $\left\{F_{\alpha}: S \rightarrow X_{\alpha}\right\}$ that map $S$ onto $\mathcal{S}$.

(B) There is a closed subspace $W$ of a Cantor space and a family of continuous surjections $\left\{f_{\alpha}: W \rightarrow X_{\alpha}\right\}$ that map $W$ onto $\delta$.

Proof. Clearly (B) implies (A). Assume (A), and consider the set

$$
Y=\left\{\left\langle F_{\alpha}(s)\right\rangle \mid s \in S\right\} \subset \prod_{\alpha} X_{\alpha} .
$$

Received by the editors December 26, 1974 and, in revised form, May 28, 1975.

AMS (MOS) subject classifications (1970). Primary 54C05, 54B25; Secondary 54C60.

Key words and phrases. Inverse limit spectra, Cantor spaces, continuous surjections. 
For any $y=\left\langle F_{\alpha}(s)\right\rangle$ in $Y$ and any pair $\alpha<\beta$, we have

$$
p_{\alpha}(y)=F_{\alpha}(s)=f_{\alpha \beta}\left(F_{\beta}(s)\right)=f_{\alpha \beta}\left(p_{\beta}(y)\right),
$$

so that $Y \subset X_{\infty}$. Since each $X_{\alpha}$ is compact Hausdorff, it follows that $\bar{Y}$ is a compact Hausdorff subspace of $X_{\infty}$; thus there is a Cantor space $K$ with closed subspace $W$ and a continuous surjection $f: W \rightarrow \bar{Y}$. For each $\alpha$ define $f_{\alpha}: W \rightarrow X_{\alpha}$ by $f_{\alpha}=p_{\alpha} \circ f$, where $p_{\alpha}$ is the restriction to $\bar{Y}$ of the $\alpha$ th projection map in $\Pi_{\alpha} X_{\alpha}$. Each $f_{\alpha}$ is a continuous surjection, being the composition of two such maps. In addition, for each pair $\alpha<\beta$,

$$
f_{\alpha}=p_{\alpha} \circ f=\left(f_{\alpha \beta} \circ p_{\beta}\right) \circ f=f_{\alpha \beta} \circ\left(p_{\beta} \circ f\right)=f_{\alpha \beta} \circ p_{\beta},
$$

completing the proof.

In view of the above, we will say $\delta$ can be mapped onto if condition (A) holds. The topological structure of the spaces that can map onto $\delta$ will be determined by the structures of the $\left\{X_{\alpha}\right\}$ and the cardinality of the index set $Q=\{\alpha\}$.

\section{Specific types of inverse spectra.}

EXAMPLE. Let $\delta$ be the inverse spectrum defined schematically in Figure 1, pointwise in Figure 2. Clearly $X_{\infty}=\varnothing$, so $\delta$ cannot be mapped onto.

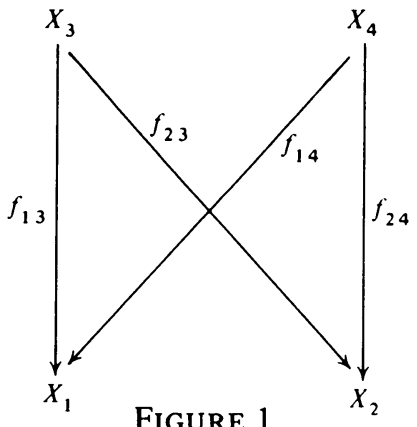

FIGURE 1

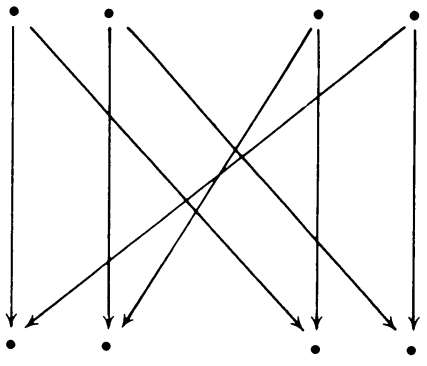

FIGURE 2

The difficulties with this inverse spectrum arise from the fact that the index set $\mathbb{Q}=\{1,2,3,4\}$ is not directed (no element of $\mathscr{Q}$ follows both 3 and 4 ) but is partially directed ( 1 and 2 are unrelated, but each of 3 and 4 follows both). In the case of either extreme-the index set $Q=\{\alpha\}$ is directed, or $Q$ is not partially directed-we will show $\delta$ can be mapped onto. The former case follows easily from known results. The latter is new, and requires some development.

THEOREM 2. Let $\delta=\left\{X_{\alpha} ; f_{\alpha \beta}\right\}$ be an inverse spectrum of compact Hausdorff spaces where

(a) each $f_{\alpha \beta}$ is a continuous surjection,

(b) the index set $Q=\{\alpha\}$ is directed.

Then $\delta$ can be mapped onto.

Proof. In view of (b) (see [1]),

$$
p_{\alpha}\left(X_{\infty}\right)=\bigcap_{\beta>\alpha}\left(f_{\alpha \beta}\left(X_{\beta}\right)\right) .
$$


From (a), each map thus defined is surjective: thus the family $\left\{\left.p_{\alpha}\right|_{X_{\infty}}\right\}$ maps $X_{\infty}$ onto $\delta$.

Definition 2. Let $\delta$ be an inverse spectrum, with $<$ the partial order on the index set $\mathbb{Q}=\{\alpha\}$. If

$$
\alpha \nless \beta \text { and } \beta<\gamma \text { imply } \alpha \nless \gamma
$$

we say $\delta$ is tree-like. If in addition, $\mathscr{Q}$ has a unique minimal element, we say $\delta$ is a tree.

Every tree-like inverse spectrum $\delta$ can canonically be embedded in a tree $\mathcal{T}=\mathcal{T}(\delta)$. One need only adjoin a distinguished element $*$ to the index set $\mathbb{Q}=\{\alpha\}$ and set $*<\alpha$ for each $\alpha \in \mathbb{Q}$. Let $X_{*}=\left\{x_{*}\right\}$ be a one-point space, and set $f_{* \alpha}(x)=x_{*}$ for each $x$ in each $X_{\alpha}$. The spectrum $\mathcal{T}=\left\{\left\{X_{\alpha}\right\} \cup X_{*}\right.$; $\left.\left\{f_{\alpha \beta}\right\} \cup\left\{f_{* \alpha}\right\}\right\}$ is a tree. Clearly $\delta$ can be mapped onto if and only if $\mathscr{T}$ can be mapped onto.

THEOREM 3. Let $\mathcal{T}=\left\{X_{\alpha} ; f_{\alpha \beta}\right\}$ be a tree of compact Hausdorff spaces, where each $f_{\alpha \beta}$ is a continuous surjection. Then $\tau$ can be mapped onto.

Proof. We will show by transfinite induction that each $\left.p_{\alpha}\right|_{X_{\infty}}: X_{\infty} \rightarrow X_{\alpha}$ is surjective; i.e., given $x_{\alpha_{0}} \in X_{\alpha_{0}}$ there is a point $y=\left\langle y_{\alpha}\right\rangle \stackrel{\infty}{\in} X_{\infty}$ with $y_{\alpha_{0}}$ $=x_{\alpha_{0}}$.

Let $\ll$ be a well-ordering of $\mathscr{Q}=\{\alpha\}$, and denote by $P(\alpha)$ the proposition

$y_{\beta}$ has been designated for all $\beta<\alpha$;

$$
\text { and if } \gamma<\beta<\alpha \text {, then } f_{\gamma \beta}\left(y_{\beta}\right)=y_{\gamma} \text {. }
$$

Begin the induction by setting $y_{\alpha_{0}}=x_{\alpha_{0}}$ and designating $y_{\gamma}=f_{\gamma \alpha_{0}}\left(y_{\alpha_{0}}\right)$ for all $\gamma<\alpha_{0}$. Now let $\alpha$ be given such that $P(\beta)$ holds for all $\beta \ll \alpha$. It may be that $y_{\alpha}$ has been designated: if $\alpha<\beta$ for some $\beta \ll \alpha$, then $(* *)$ follows trivially. Suppose then that $y_{\alpha}$ has not been designated. One sees easily from (*) of Definition 2 that

$$
\mathscr{B}=\left\{\beta \in \mathbb{Q} \mid \beta<\alpha \text { and } y_{\beta} \text { has been designated }\right\}
$$

is totally ordered by $<$. If $\gamma, \beta \in \mathscr{B}$ and $\gamma<\beta$, we have that $y_{\gamma}=f_{\gamma \beta}\left(y_{\beta}\right)$, so that

$(* * *) \quad$ If $\gamma, \beta \in \mathscr{B}, \quad \gamma<\beta$ implies $f_{\beta \alpha}^{-1}\left(y_{\beta}\right) \subset f_{\gamma \alpha}^{-1}\left(y_{\gamma}\right)$.

Each $f_{\beta \alpha}: X_{\alpha} \rightarrow X_{\beta}$ is a continuous surjection, so each $f_{\beta \alpha}^{-1}\left(y_{\beta}\right)$ is closed in $X_{\alpha}$. By $(* * *)$, the collection $\left\{f_{\beta \alpha}^{-1}\left(y_{\beta}\right) \mid \beta \in \mathscr{B}\right\}$ has the finite intersection property. Since $X_{\alpha}$ is compact Hausdorff, $\cap\left\{f_{\beta \alpha}^{-1}\left(y_{\beta}\right) \mid \beta \in \mathscr{B}\right\}$ is nonempty. Designate any point in the intersection as $y_{\alpha}$, and set $y_{\beta}=f_{\beta \alpha}\left(y_{\alpha}\right)$ for all $\beta<\alpha$. This completes the proof.

COROLlaRY 2. The following can be mapped onto.

(1) Any tree-like spectrum of compact Hausdorff spaces.

(2) Any spectrum of compact Hausdorff spaces where there is a set $\mathscr{B} \subset \mathbb{Q}$ cofinal, where $B$ is tree-like. 
COROLlARY 3. Let $\delta$ be an inverse spectrum which, in addition to satisfying any of the conditions of Corollary 2, satisfies

(i) each $X_{\alpha}$ is compact metric,

(ii) the index set $\mathbb{Q}=\{\alpha\}$ is countable.

Then the Cantor set $2^{\omega}$ maps onto $\mathcal{S}$, with each $f_{\alpha}$ continuous. In particular, $2^{\omega}$ maps continuously onto the inverse spectrum mentioned in the introduction.

The proofs of Corollaries 2 and 3 are obvious.

The author thanks the referee for suggesting he consider Theorem 1 in its full generality.

\section{REFERENCE}

1. James Dugundji, Topology, Allyn and Bacon, Boston, Mass., 1966, p. 435. MR 33 \# 1824.

Department of Mathematics, University of California, Davis, California 95616

Current address: SESAME, \% Physics, University of California, Berkeley, California 94720 